\title{
Dinâmica de regeneração de Merostachys skvortzovii Send. em remanescente de Floresta com Araucaria
}

\author{
Merostachys skvortzovii Send. regeneration dynamics in a Araucarian forest remnant
}

\author{
Raquel Rejane Bonato Negrelle ${ }^{\mathrm{I}}$ Fábio Hiratsuka Veiga ${ }^{\mathrm{II}}$
}

\section{RESUMO}

\begin{abstract}
Apresentam-se resultados de monitoramento da dinâmica de regeneração de Merostachys skvortzovii Send. (taquaralixa), englobando dados de recrutamento, crescimento e mortalidade de plântulas (Floresta Ombrófila Mista, General Carneiro, Paraná, $26^{\circ} 25^{\prime} \mathrm{S}$ e $51^{\circ} 18^{\prime} \mathrm{W}$ ). Foram avaliadas duas clareiras de tamanhos (aprox. 40mx40m) e situações de abertura do dossel similares, resultantes da morte de touceiras de taquara-lixa entre 20032005, utilizando-se 80 parcelas (1mx1m) em cada clareira (2007). Mensalmente, registrou-se a inclusão, permanência e mortalidade de plântulas de M. skvortzovii nessas parcelas. Evidenciou-se densidade média de plântulas diferenciada, mas similar padrão de distribuição espacial dessas plântulas nas clareiras avaliadas. As médias mensais de recrutamento, crescimento em altura e mortalidade foram significativamente diferentes em ambas as clareiras. Não se detectou correlação significativa entre as variáveis biológicas observadas (crescimento, recrutamento e mortalidade) e a variabilidade de pluviosidade e temperatura registradas no periodo de monitoramento. Em termos gerais, pode-se inferir que a taquara-lixa apresentava a capacidade de recolonizar as áreas abertas após o evento sincrônico de mortalidade, sendo que as características ambientais de cada clareira podem influenciar essa ocupação. A concentração das plântulas nas bordas das clareiras pode determinar a ampliação da área de ocupação da espécie no local de estudo. Entretanto, somente o monitoramento a médio e longo prazo das clareiras geradas pela morte da taquara-lixa permitirá compreender melhor o ciclo de vida dessa espécie e seu efetivo papel no fechamento dessas clareiras e dinâmica da floresta que a abriga.
\end{abstract}

Palavras-chave: Bambusoideae, floresta ombrófila mista, Poaceae, recolonização de taquara-lixa.

\section{ABSTRACT}

The results of monitoring the Merostachys skvortzovii Send. regeneration dynamics, including seedling recruitment, growth and mortality (Araucarian Forest, General Carneiro, Paraná, $26^{\circ} 25^{\prime} S$ e $51^{\circ} 18^{\prime} \mathrm{W}, 1000 \mathrm{~m}$ a.s.l.) are presented. Two gaps with similar size $(40 \mathrm{~m} \times 40 \mathrm{~m})$ and opening situation, resulting from M. skvortzovii dye-off s in 2003-2005, on which 80 small plots (1mx $1 \mathrm{~m})$ were evaluated (2007). Monthly, it were registered the inclusion, permanence and mortality of seedlings of M. skvortzovii in these plots. It was evidenced different seedling average density among gaps, but similar seedlings spatial distribution pattern in both gaps. The monthly averages of recruitment, growth in height and mortality were significantly different in both gaps. Significant correlation was not detected between the biological observed variables (growth, recruitment and mortality) and the variability of pluviosity and temperature registered in during the monitoring. In general terms, it is possible to infer that the M. skvortzovii representative were capable of re-colonizing the areas opened after the synchronous dye-off event, but the environmental characteristics of each gap can influence this occupation. The concentration of the seedlings in the edges of the gaps can determine the enlargement of the species occupation area. Meantime, only middle to long term monitoring of the clearings produced by the M. skvortzovii dyeoff will allow the understanding of the this species life cycle and its effective paper in the closure of these gaps as well as in the dynamic of the forest that shelters it.

Key words: bamboo re-colonization, Bambusoideae, Mixt Ombrophilous Forest, Poaceae.

\section{INTRODUÇÃO}

Os bambus e taquaras compreendem o maior e mais bem sucedido grupo de gramíneas encontrado nas florestas tropicais (SENDULSKY, 1997). São vegetais semelparos, isto é, apresentam florescimento que varia de três a 120 anos, com

'Laboratório OIKOS, Departamento de Botânica, Universidade Federal do Paraná (UFPR), CP 19031, 81531-990, Curitiba, PR, Brasil.

E-mail: negrelle@ufpr.br. Autor para correspondência.

IIPrograma de Pós-graduação em Geociências, Instituto de Geociências, Universidade Federal do Rio Grande do Sul (UFRGS), Porto Alegre, RS, Brasil. 
floração e frutificação maciças entre todos os indivíduos da espécie (JANZEN, 1976; WIDMER, 1998). Produzem grandes quantidades de sementes até a morte sincronizada de todos os indivíduos (JANZEN, 1976). Após a floração simultânea, a regeneração das touceiras dependerá totalmente do estabelecimento e crescimento das plântulas de bambu que emergem no ano seguinte (LI et al., 2006). A resposta integrada de ecossistemas florestais a esses eventos reprodutivos de bambus ainda não está bem entendida, devido, em parte, ao seu ciclo de vida longevo e particular e a sua complexa interação com múltiplos processos ecossistêmicos (MONTTI et al., 2011a). Entretanto, é reconhecido que o tempo necessário à recuperação das populações de bambus, após a sua mortalidade sincrônica, é um importante determinante da dinâmica das florestas onde esses bambus estão presentes (JUDZIEWICZ et al., 1999).

No Brasil, há registros de diversas espécies de bambus, tanto nativos quanto exóticas. Dentre as espécies nativas, dispersas ao longo de todas as regiões brasileiras, destacam-se as popularmente conhecidas por taquara, taboca, jativoca, taquaruçu ou taboca-açu, conforme sua região de ocorrência. No sul do Brasil, os gêneros mais representativos são Merostachys, Guadua e Chusquea. O gênero Merostachys, conhecido vulgarmente como taquara, distribui-se desde a América Central até a Argentina, com o centro de diversidade no Brasil (SENDULSKY, 1992; SODERSTROM et al., 1998; WATSON \& DALLWITZ, 2011). Das espécies brasileiras, 87\% encontram-se distribuídas nas florestas montanas do leste e sudeste brasileiro e desempenham um papel ecológico importante nos ecossistemas em que se encontram, devido à sua influência sob a regeneração de espécies arbóreas (FARRELLY, 1984; SENDULSKY, 1995; LONDOÑO, 2001; QUATTROCCHI, 2006).

Dentre essas espécies de Merostachys que ocorrem no Brasil, em 2003, registrou-se o início de massivo florescimento e consequente mortalidade de touceiras de M. skvortzovii Send. em diversas localidades do sul do estado do Paraná e norte de Santa Catarina em região de Floresta Ombrófila Mista. Coleções conhecidas dessa espécie, de 1941, 1972-1974, sugeriam um ciclo de florescimento de 31-33 anos (SENDULSKY, 1995; SCHWARZBACH et al., 2008). Devido a esse longo intervalo de tempo, há poucos registros sobre comportamento dessa espécie após a floração sincrônica. De acordo com legislação do Estado de São Paulo, essa espécie está incluída na lista de espécies da flora ameaçadas de extinção (SÃO PAULO, 2004).
Dessa forma, organizou-se o trabalho aqui apresentado de maneira a aproveitar o fenômeno biológico raro, de floração e frutificação maciças de M. skvortzovii em área de Floresta Ombrófila Mista. Especificamente, objetivou-se monitorar e avaliar a dinâmica de regeneração de $\boldsymbol{M}$. skvortzovii a partir da quantificação da densidade de suas sementes no solo, associado ao recrutamento, padrão de distribuição, crescimento e mortalidade de plântulas. Avaliou-se também a possibilidade de influência das condições ambientais (pluviosidade e temperatura) sobre a dinâmica de regeneração de plântulas. Os resultados obtidos foram confrontados ao já reportado para a espécie e grupo taxonômico na literatura.

\section{MATERIAL E MÉTODOS}

O estudo foi desenvolvido em remanescente de Floresta Ombrófila Mista (Indústrias Pedro N. Pizzatto, General Carneiro, Paraná; $26^{\circ} 25^{\prime} \mathrm{S}$ e $51^{\circ}$ $18^{\prime} \mathrm{W} ; 1000 \mathrm{~m}$ s.n.m.). Essa área engloba três tipos de solos: Cambissolo Álico, Associação solos Litólicos Álicos + Terra Rocha Estruturada Álica e Associação Terra Bruna Estruturada Álica + Cambissolo Álico (EMBRAPA, 1984). O clima dessa região, segundo a classificação de Köppen, é caracterizado como Subtropical Úmido Mesotérmico $(\mathrm{Cfb})$, cuja temperatura média é inferior a $22^{\circ} \mathrm{C}$ durante o mês mais quente, com verões frescos, invernos com ocorrência de geadas e sem estação seca (PARANÁ, 1987). A cobertura vegetal original dessa área é classificada como Floresta Ombrófila Mista, também conhecida como Floresta com Araucária. Devido às diversas atividades antrópicas, essa cobertura vegetal encontrase bastante reduzida, apresentando fragmentos em diferentes estádios sucessionais, intercalados por áreas de reflorestamento, principalmente de pinus, araucária e erva-mate (IBGE, 1992).

Para a realização desta pesquisa, selecionaram-se duas clareiras que apresentavam tamanhos (aprox. 40mx40m) e situações de abertura do dossel similares, resultantes da morte de touceiras de taquara-lixa entre 2003-2005. O monitoramento da dinâmica de regeneração das plântulas ocorreu entre março e setembro de 2007. Para tanto, em cada clareira foi escolhido um ponto central de onde foram lançados dois transectos nos sentidos norte-sul e leste-oeste. Sobre esses transectos, foram alocadas 40 parcelas de $1 \mathrm{mx} 1 \mathrm{~m}$, totalizando 80 parcelas em cada área. No censo inicial, registrou-se a presença, localização e mensurou-se a altura de todos os colmos vivos (plântulas) que haviam se desenvolvido na clareira após o evento de morte da touceira 
original. Na sequência, mensalmente, registrouse o recrutamento (inserção de novos indivíduos), crescimento em altura e mortalidade de plântulas de M. skvortzovii nas parcelas. A altura foi medida com régua graduada a partir do ponto de inserção do colmo no solo. A homogeneidade das amostras foi avaliada a partir da comparação entre as variâncias das médias mensais obtidas para as variáveis consideradas, utilizando-se o Teste F em nível de 2,5\%, conforme detalhado em BEIGUELMAN (202). O padrão espacial dos indivíduos amostrados foi avaliado pelo índice de dispersão de Morisita (Id), como proposto por KREBS (1999), em associação à frequência de ocorrência da espécie nas parcelas amostradas.

De modo a não interferir na dinâmica de estabelecimento de plântulas, a análise do banco de sementes no solo foi realizada em outra clareira próxima e similar às avaliadas para dinâmica de regeneração de plântulas. Nesta, foram amostrados 12 pontos equidistantes em cada um dos eixos similares aos estabelecidos nas parcelas anteriormente descritas $(n=24)$. Para a coleta de solo, utilizou-se anel de metal $(0,5 \mathrm{mx} 0,5 \mathrm{~m})$, englobando-se a serapilheira e a camada do solo imediatamente inferior até a profundidade de $0,5 \mathrm{~m}$ (outubro/2006). As amostras coletadas foram acondicionadas em sacos de papel e transportadas para a estufa do laboratório OIKOS na Universidade Federal do Paraná, onde foram pesadas antes e depois da secagem. O material seco foi destorroado em peneira (malha $6 \mathrm{~mm}$ ), para quantificação das sementes de M. skvortzovii, com auxílio de lupa e pinça. A quantidade média de sementes obtida, considerando os 24 pontos amostrais $\left(6 \mathrm{~m}^{2}\right)$, foi extrapolada para número de sementes por hectare.

Mensalmente, obteve-se, junto ao SIMEPAR, a média pluviométrica e de temperatura registradas pela estação meteorológica mais próxima da área de estudo, localizada no município de União da Vitória. A influência das médias climáticas sobre o crescimento, recrutamento e mortalidade de plântulas, foi avaliada pelo coeficiente de correlação de Spearman. Nessa análise, sabendo que algumas plantas apresentam respostas fenológicas atrasadas a determinado estímulo ambiental (MARQUES \& OLIVEIRA, 2004), utilizaram-se dados retroativos em até três meses do início do monitoramento.

\section{RESULTADOS E DISCUSSÃO}

Registrou-se um total de 124 sementes de M. skvortzovii em $6 \mathrm{~m}^{2}\left(\mathrm{n}=24 \mathrm{x} 0,25 \mathrm{~m}^{2}\right)$, o que representaria um total de 207 mil sementes ha-1 ${ }^{-1}$ Em média, evidenciaram-se $5,17 \pm 4,50$ sementes por unidade amostral. Observou-se grande variabilidade na quantidade de sementes por unidade amostral, evidenciando-se densidade crescente do centro em direção ao limite externo (borda) da clareira. No total, foram registradas 544 plântulas de M. skvortzovii $\left(\mathrm{N}_{\mathrm{A}}=144 ; \mathrm{N}_{\mathrm{B}}=400 ; 80 \mathrm{~m}^{2}\right)$, o que corresponde a 34 mil plântulas ha-1 $\left(\mathrm{N}_{\mathrm{A}}=18 \mathrm{mil} \mathrm{ha}^{-1}\right.$; $\mathrm{N}_{\mathrm{B}}=50 \mathrm{mil} \mathrm{ha}^{-1}$ ) (Figura 1). A densidade média de plântulas por parcelas foi distinta entre as clareiras avaliadas $\left(\mathrm{DM}_{\mathrm{A}=} 1,86 \pm 2,71\right.$ plântulas $\mathrm{m}^{-2} ; \mathrm{s}^{2}=7,36$; $\mathrm{DM}_{\mathrm{B}=} 5,00 \pm 4,93$ plântulas $\mathrm{m}^{-2} ; \mathrm{s}^{2}=24,35$ ) (Teste $\left.\mathrm{F}_{2,5 \%}\right)$. A frequência absoluta de plântulas nas parcelas também foi distinta entre essas $\left(\mathrm{FA}_{\mathrm{A}}=50 \%\right.$; $\mathrm{FA}_{\mathrm{B}}=92,5 \% ; \mathrm{n}=80$ parcelas $)$. Em ambas as clareiras, observou-se distribuição agrupada dos indivíduos amostrados $\left(\operatorname{Id}_{\mathrm{A}}=2,55 ; \quad \mathrm{Id}_{\mathrm{B}}=1,77\right)$, com padrão crescente do centro em relação à borda da clareira.

As médias mensais de recrutamento $\left(\mathrm{x}_{\mathrm{A}}=10,71 \pm 8,24\right.$ plântulas; $\mathrm{s}^{2}=67,90 ; \mathrm{x}_{\mathrm{B}}=11,67 \pm 3,20$; $\left.\mathrm{s}^{2}=10,27\right)$, assim como de crescimento em altura $\left(\mathrm{x}_{\mathrm{A}}=0,72 \pm 0,18 \mathrm{~cm} ; \quad \mathrm{s}^{2}=0,03 ; \quad \mathrm{x}_{\mathrm{B}}=0,74 \pm 0,13 \mathrm{~cm} ;\right.$ $\left.\mathrm{s}^{2}=0,02\right)$ e mortalidade $\left(\mathrm{x}_{\mathrm{A}}=4,14 \pm 1,21\right.$ plântulas; $\left.s^{2}=1,48 ; \quad x_{B}=20 \pm 6,87 ; \quad s^{2}=47,20\right)$, foram também significativamente diferentes em ambas as clareiras (Teste $\mathrm{F}_{2,5 \%}$ ) (Figura 1). Registrou-se crescimento populacional diferenciado em ambas as clareiras. $\mathrm{Na}$ clareira A, evidenciou-se, ao longo do monitoramento, taxa decrescente de inclusão de plântulas, com taxa de mortalidade mensal razoavelmente homogênea. $\mathrm{Na}$ clareira B, evidenciou-se taxa crescente de inclusão de plântulas e taxas mensais de mortalidade mais altas e heterogêneas, comparativamente à clareira $\mathrm{A}$ (Figura 1). Não se detectou correlação significativa entre as variáveis biológicas observadas (crescimento, recrutamento e mortalidade) e a variabilidade de pluviosidade e temperatura registradas no período de monitoramento ou mesmo retroativa em até três meses antes deste.

$\mathrm{O}$ arranjo das densas touceiras com colmos pendentes, com inflorescências terminais e arqueados em direção oposta ao centro da touceira parece ser o principal determinante do padrão de distribuição de sementes no solo, registrado para $\boldsymbol{M}$. skvortzovii. Esse padrão, com densidade crescente em direção à borda da clareira, também foi descrito para outras espécies de bambus (OLMOS, 1990; GUILHERME \& RESSEL, 2001). No entanto, a quantidade de sementes registrada nos bancos de sementes das duas clareiras avaliadas foi bastante inferior à potencial produção de sementes descrita para bambus e taquaras. GUILHERME \& RESSEL (2001) estimaram produção para Merostachys 


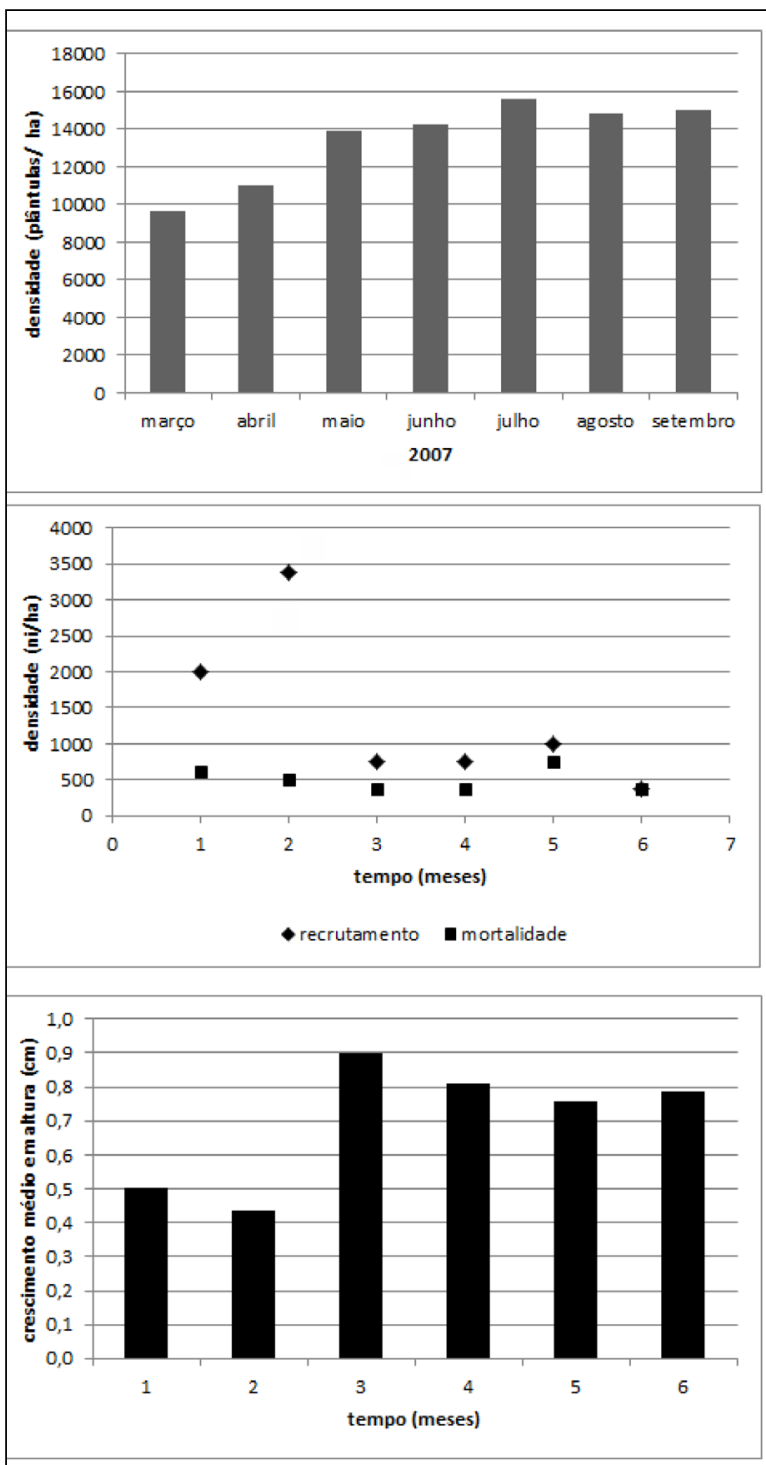

clareira A
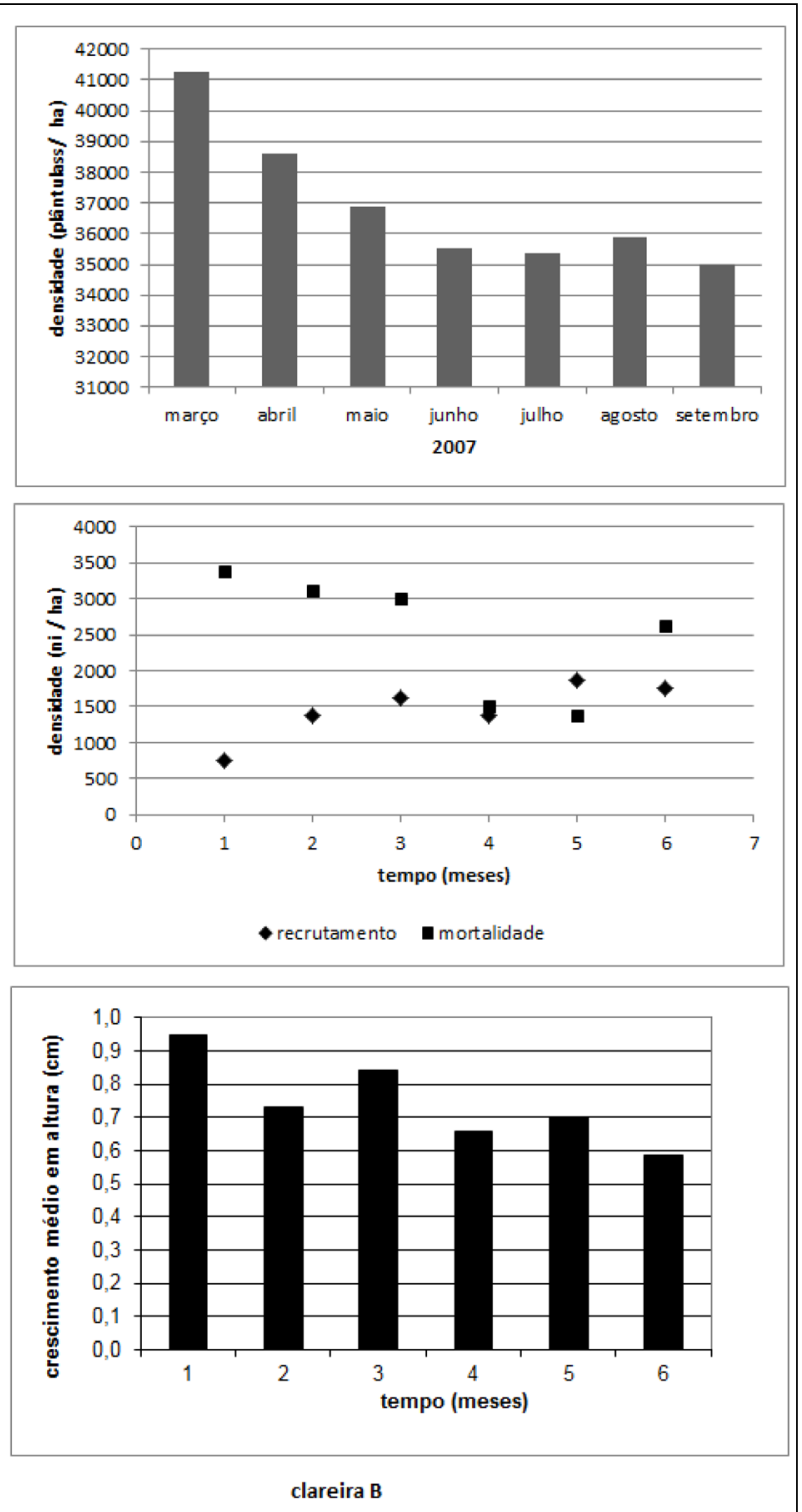

Figura 1 - Densidade total, recrutamento, mortalidade e crescimento de plântulas registradas no monitoramento de regeneração de $\boldsymbol{M}$. skvortzovii, em duas áreas amostrais (General Carneiro, Paraná, março-setembro/07).

riedeliana em mais de 30 milhões de sementes/ ha $^{-1}$ e GONZÁLEZ \& DONOSO (1999) estimaram produção para Chusquea quila em mais de 195 milhões de sementes ha ${ }^{-1}$. Há também registros de Merostachys spp., produzindo camadas de sementes com 12 a $15 \mathrm{~cm}$ de espessura (OLMOS, 1990).

Um dos motivos que pode ter determinado a baixa densidade registrada na área estudada pode ter sido a alta mortalidade devido às baixas temperaturas do inverno, após a floração, dado que as sementes de espécies gregárias de bambus tropicais e subtropicais não apresentam dormência (BELLAIRS et al., 2008).
LI et al. (2006) observou uma mortalidade de até 85\% nas sementes do bambu Fargesia murieliae, entre um inverno e outro. Outro fator que pode ter determinado essa baixa densidade é a predação das sementes, principalmente devido ao fenômeno das "ratadas". Após a frutificação sincrônica, as touceiras de Merostachys spp. produzem grandes quantidades de sementes (OLMOS, 1996; OLMOS et al., 1993), aumentando a disponibilidade de alimento. Esse processo não foi diretamente verificado na área estudada. Entretanto, registrou-se, durante 2005, aumento no número de ratos silvestres (Oligoryzomys 
spp. e Akodon spp.) em outras regiões do Paraná, com alta incidência de $\boldsymbol{M}$. skvortzovii (LIEBSCH \& REGINATO, 2009). Esse crescimento na população de roedores após o evento de floração já havia sido também documentado por PEREIRA (1941) e GIOVANNONI et al. (1946), em regiões próximas à área estudada. CESTARI \& BERNARDI (2011) também relatam que a intensa atividade predatória de aves, concomitante à dispersão de sementes afeta incisivamente e negativamente sobre a dinâmica de regeneração M. neesii na Floresta Atlântica.

Esperava-se que a dinâmica de regeneração das plântulas tivesse alguma correlação com a variação regional de pluviosidade e temperatura, uma vez que, tanto a germinação quanto o crescimento, são geralmente afetados pelas condições ambientais (CATOVSKY \& BAZZAZ, 2000). Contrariamente ao esperado, essa relação não foi evidenciada na área estudada. Entretanto, a diferença observada no que concerne ao recrutamento, crescimento e mortalidade de plântulas em ambas as clareiras explicita que fatores microambientais (luz, umidade do solo, p.ex.) podem ser preponderantes para determinar o sucesso ou não do recrutamento $\mathrm{e}$ estabelecimento das plântulas de $\boldsymbol{M}$. skvortzovii. As clareiras geradas pela morte de bambus e taquaras são espaços de alta complexidade físico-estrutural, configurados por grande amplitude de formas, tamanhos e orientação da entrada de luz, que resultam em elevada complexidade microambiental (BUDKE et al., 2010). Para muitas espécies, o recrutamento e estabelecimento de plântulas são diretamente dependentes dessas condições microambientais específicas (BROWN, 1993).

Por outro lado, a maior densidade de plântulas, devido ao microambiente favorável, parece determinar valores mais altos de mortalidade, resultada de controle populacional denso-dependente, como o observado para na clareira B (GUNTON \& KUNIN, 2009). No entanto, apesar do crescimento populacional diferenciado, em ambas as clareiras estudadas, registrou-se efetiva capacidade de produção e manutenção de estoque de plântulas de M. skvortzovii, promovendo a sua permanência na área. Como esperado, identificou-se, para as plântulas padrão, similar ao detectado para as sementes, com maior densidade destas na borda das clareiras. Esse grupo de plantas se espalha rapidamente através de rizomas paquimorfos, formando densas touceiras com profusão de colmos pendentes (CAMPANELLO et al., 2007). Dada a sua localização mais densa nos limites externos da clareira, uma das implicações possíveis desse padrão de recolonização e crescimento seria o aumento da área de ocupação atual da espécie, resultado em um ciclo de distúrbio autoperpetuado. Como consequência possível dessa dinâmica, cita-se a não facilitação do estabelecimento de plântulas de espécies arbóreas (MONTTI, 2011b) e consequente redução na densidade e área basal de espécies arbóreas que coexistem com a taquara (GRISCOM \& ASHTON, 2006), determinando efeitos em longo prazo sobre a estrutura e funcionamento do ecossistema florestal.

\section{CONCLUSÃO}

Em termos gerais, pode-se inferir que a taquara-lixa apresentava a capacidade de recolonizar as áreas abertas após o evento sincrônico de mortalidade, sendo que as características de cada clareira podem influenciar essa ocupação. A concentração das plântulas nas bordas das clareiras pode determinar a ampliação da área de ocupação da espécie no local de estudo. Entretanto, somente o monitoramento a médio e longo prazo das clareiras geradas pela morte da taquara-lixa permitirá compreender melhor o ciclo de vida dessa espécie e seu efetivo papel no fechamento dessas clareiras e dinâmica da floresta que a abriga.

\section{AGRADECIMENTOS}

Às Indústrias Pedro N. Pizzatto, General Carneiro, Paraná, pela disponibilidade da área para a pesquisa e apoio logístico.

\section{REFERÊNCIAS}

BEIGUELMAN, B. Curso prático de bioestatística. Natal: FUNPEC, 2002. 254p. BELLAIRS et al. A tropical, gregariously semelparous bamboo shows no seed dormancy. Biotropica, Malden, v.40, n.1, p.28-31, 2008. Disponível em: <http://onlinelibrary. wiley.com/doi/10.1111/j.1744-7429.2007.00336.x/pdf $>$. Acesso em: 5 dez. 2012. doi: 10.1111/j.1744-7429.2007.00336.x.

BROWN, N. The implications of climate and gap microclimate for seedling growth conditions in a Bornean lowland rain forest. Journal of Tropical Ecology, Cambridge, v.9, n.2, p.153-168, 1993. Disponível em: <http://journals.cambridge.org/action/ displayIssue decade $=1990 \& \mathrm{jid}=$ TRO\&volumeId=9\&issueId $=02 \&$ iid $=5247684>$. Acesso em: 5 dez. 2011. doi: 10.1017/ S0266467400007136.

BUDKE, J.C. et al. Bamboo dieback and tree regeneration responses in a subtropical forest of South America. Forest Ecology and Management, Fort Collins, v.260, n.1-3, p.1345-1349, 2010. Disponível em: <http://ac.els-cdn.com/S0378112710004123/1s 2.0-S0378112710004123-main.pdf?_tid=b062b3ae9b92-11e2-86fb-00000aab0f27\&acdnat $=1364906690$ ac73edd8abb6ec218c1201b56482ee47>. Acesso em: 2 dez. 2011. doi: 10.1016/j.foreco.2010.07.028. 
CAMPANELLO, P.I. et al. Tree regeneration and microclimate in a liana and bamboo-dominated semideciduous Atlantic Forest. Forest Ecology and Management, Fort Collins, v.252, n.1-3, p.108-117, 2007. Disponível em: $<$ http://ac.els-cdn.com/S0378112707004744/1 s2 .0-S $0378112707004744-\mathrm{main} . p d f ?$ tid=034d46249b93-11 e2-ba39-00000aab0f6b\&acdnat $=1364906829$ b9e6e5b56951a0f0509a443e724e58bf>. Acesso em: 5 dez. 2011. doi: 10.1016/j.foreco.2007.06.032.

CATOVSKY, S.; BAZZAZ, F.A. The role of resource interactions and seedling regeneration in maintaining a positive feedback in hemlock stands. Journal of Ecology, London, n.88, p.100112, 2000. Disponível em: <http://onlinelibrary.wiley.com/ doi/10.1046/j.1365-2745.2000.00428.x/pdf>. Acesso em: 25 nov. 2011. doi: 10.1046/j.1365-2745.2000.00428.x.

CESTARI, C.; BERNARDI, C.J. Predation of the buffy-fronted seedeater Sporophila frontalis (Aves: Emberizidae) on Merostachys neesii (Poaceae: Babusoideae) seeds during a masting event in the Atlantic forest. Biota Neotroprica, Campinas, v.11, n.3, n.p., 2011. Disponível em: $<$ http://www. biotaneotropica.org.br/v11n3/pt/fullpaper?bn00711032011+en>. Acesso em: 22 nov. 2011. doi: 10.1590/S1676-06032011000300033.

EMBRAPA (EMPRESA BRASILEIRA DE PESQUISA AGROPECUÁRIA). Levantamento de reconhecimento dos solos do Estado do Paraná. Curitiba: EMBRAPA-SNLCS/ SUDESUL/IAPAR, 1984. 414p.

FARRELLY, D. The book of bamboo. San Francisco: Sierra Club Books, 1984. 352p.

GIOVANNONI, M. et al. Sobre as "ratadas" do primeiro planalto paranaense. Arquivos de Biologia e Tecnologia, Curitiba, n.1, p.185-195, 1946

GONZÁLEZ, M.E.; DONOSO, C. Producción de semillas y hojarasca en Chusquea quila (Poaceae: Bambusoideae), posterior a su floración sincrónica en la zona centro-sur de Chile. Revista Chilena de Historia Natural, Santiago, v.72, n.2, p.169-180, 1999.

GRISCOM, B.W.; ASHTON, P.MS. A self-perpetuating bamboo disturbance cycle in a neotropical forest. Journal of Tropical Ecology, Cambridge, v.22, n.5, p.587-597, 2006. Disponível em: $\quad<$ http://journals.cambridge.org/download.php?file $=\% 2 \mathrm{~F} 122$ 2_57703FBB9102813FCB04E4161B314297_journals_TRO TRO22 05 S0266467406003361a.pdf\&cover $=$ Y \& co $\overline{d e}=93 \mathrm{e} \overline{9}$ $4 \mathrm{fb} 2382 \mathrm{~b} 39 \overline{3} \mathrm{~d} 4579326 \mathrm{df0a} 7 \mathrm{aa} 58>$. Acesso em: 22 nov. 2011 doi:10.1017/S0266467406003361.

GUILHERME, F.A.G.; RESSEL, K. Biologia floral e sistema de reprodução de Merostachys riedeliana (Poaceae: Bambusoideae). Revista Brasileira de Botânica, São Paulo, v.24, n.2, p.205-211, 2001.

GUNTON, R.M.; KUNIN, W.E. Density-dependence at multiple scales in experimental and natural plant populations. Journal of Ecology, Londres, v.97, n.3, p.567-580, 2009. Disponível em: <http://onlinelibrary.wiley.com/doi/10.1111/ j.1365-2745.2007.01226.x/full>. Acesso em: 2 dez. 2011. doi: 10.1111/j.1365-2745.2009.01492.x.

IBGE (INSTITUTO BRASILEIRO DE GEOGRAFIA E ESTATÍSTICA). Manual técnico da vegetação brasileira. Rio de Janeiro: DEDIT/CDDI, 1992. 91p.

JANZEN, D.H. Why bamboos wait so long to flower? Annual Review of Ecology and Systematics, Palo Alto, v.7, p.347-
391, 1976. Disponível em: <http://www.annualreviews.org/doi/ pdf/10.1146/annurev.es.07.110176.002023>. Acesso em: 2 nov. 2011. doi: 10.1146/annurev.es.07.110176.002023.

JUDZIEWICZ, E.J. et al. American bamboos. Brittonia, New York, v.51, n.2, p.158, 1999. Disponível em: $<$ http://link.springer. com/content/pdf/10.2307\%2F2666622>. Acesso em: 23 nov. 2011. doi: $10.2307 / 2666622$.

KREBS, C.J. Ecological methodology. New York: Library of Congress, 1999. 620p.

LI, D.Z. et al. Simultaneous flowering of umbrella bamboo (Fargesia murieliae) at its native home in Central China. Journal of Forestry Research, Beijing, v.17, n.4, p.293-297, 2006. Disponível em: $<$ http://www.paper.edu.cn/.../1007-662X(2006)04-0293>. Acesso em: 18 nov. 2011. doi: 10.1007/s11676-006-0067-4.

LIEBSCH, D.; REGINATO, M. Florescimento e frutificação de Merostachys skvortzovii Sendulsky (taquara-lixa) no estado do Paraná. Iheringia, Série Botânica, Porto Alegre, v.64, n.1, p.5356, 2009. Disponível em: <http://www.fzb.rs.gov.br/publicacoes/ iheringia-botanica/Ih64-1-p053-056.pdf>. Acesso em: 26 set. 2011.

LONDOÑO, X. Evaluation of bamboo resource in Latin America. Beijing: INBAR, 2001. 30p.

MARQUES, M.C.M.; OLIVEIRA, P.E.A.M. Fenologia de espécies do dossel e do sub-dossel de duas Florestas de Restinga na Ilha do Mel, sul do Brasil. Revista Brasileira de Botânica, São Paulo, v.27, n.4, p.713-723, 2004. Disponível em: <http://www.scielo.br/scielo.php?script=sci_arttext\&pid $=\mathrm{S} 0100-84042004000400011 \mathrm{P}$. Acesso em: 16 nov. 2011. doi: 10.1590/S0100-84042004000400011.

MONTTI, L. et al. Flowering, died-back and recovery of semelparous woody bamboo in the Atlantic Forest. Acta Oecologica, Paris, v.37, n.4, p.361-368, 2011a. Disponível em: <http:/ac.els-cdn.com/ S1146609X1100066X/1-s2.0-S1146609X1100066X-main.pdf? tid=ac4600da-9b99-11e2-b859-00000aab0f6c\&acdnat=1364909689 ae441f93f74c253fc24bbe74c0bea50e>. Acesso em: 18 nov. 2011. doi: 10.1016/j.actao.2011.04.004.

MONTTI, L. et al. Understory bamboo flowering provides a very narrow light window of opportunity for canopy-tree recruitment in a neotropical forest of Misiones, Argentina. Forest Ecology and Management, Fort Collins, v.262, n.8, p.1350-1369, $2011 \mathrm{~b}$. Disponível em: <http://ac.els-cdn.com/S0378112711003914/1s2.0-S0378112711003914-main.pdf?_tid=f27f87b0-9b99-11e2aaf7-00000aab0f01 \&acdnat $=1364909807 \quad 5 \mathrm{f} 74 \mathrm{~d} 926 \mathrm{~d} 0389 \mathrm{e} 66 \mathrm{~d}$ 641190bdd43d072>. Acesso em: $02 \mathrm{dez}, 2011$. doi: 10.1016/j. foreco.2011.06.029.

OLMOS, F. Frutificação de Chusquea meyeriana Rupr. (Poaceae, Bambusoideae) e dinâmica populacional de aves granívoras e roedores em área de Mata Atlântica. Campinas: UNICAMP, 1990. 71p.

OLMOS, F. Satiation or deception? Mast-seeding Chusquea bamboos, birds and rats in the Atlantic Forest. Revista Brasileira de Biologia, São Carlos, v.56, p.391-401, 1996.

OLMOS, F. et al. Habits of the southern bamboo rat Kannabateomys amblyonyx (Rodentia, Echimyidae) in Southeastern Brazil. Mammalia, Paris, v.57, p.325-333, 1993. Disponível em: <http:// www.degruyter.com/dg/viewarticle/j\$002fmamm.1993.57.issue- 
3\$002fmamm.1993.57.3.325\$002fmamm.1993.57.3.325.xml;jse ssionid $=6$ C89A00CF9592DC89AA00E736F5C7D21>. Acesso em: 5 nov. 2011. doi: 10.1515/mamm.1993.57.3.325.

PARANÁ. Secretaria de Estado de Agricultura e Abastecimento. Instituto de Terras, Cartografia e Florestas. Atlas do Estado do Paraná. Curitiba, 1987. 73p.

PEREIRA, C. Sobre as "ratadas" no sul do Brasil e o ciclo vegetativo das taquaras. Arquivos do Instituto de Biologia de São Paulo, São Paulo, n.12, p.175-195, 1941.

QUATTROCCHI, U. CRC world dictionary of grasses: common names, scientific names, eponyms, synonyms and etymology. Boca Raton: CRC, 2006. 640p.

SÃO PAULO. Secretaria de Meio Ambiente. Resolução SMA 48: lista oficial das espécies da flora do Estado de São Paulo ameaçadas de extinção. 22 de setembro de 2004. 24p.

SCHWARZBACH, L.L.C. et al. Merostachys skvortzovii Sendulsky: aspectos botânicos e ecológicos - uma revisão. Visão Acadêmica, Curitiba, v.9, n.2, p.7-12, 2008. Disponível em: $<$ http://www.visaoacademica.ufpr.br/v9n2/ARTIGO01.pdf $>$. Acesso em: 25 nov. 2011.

SENDULSKY, T. Merostachys burmani (Poaceae: Bambusoideae: Bambuseae), a new species from Brazil. Novon, Saint Louis, v.2 p.111-113, 1992.
SENDULSKY, T. Merostachys multiramea (Poaceae: Bambusoideae: Bambuseae) and similar species from Brazil. Novon, Saint Louis, v.5 p.76-96, 1995.

SENDULSKY, T. Twelve new species of Merostachys (Poaceae: Bambusoideae: Bambuseae) from Brasil. Novon, Saint Louis, v.7, p.285-307, 1997. Disponível em: <http://www.jstor.org/dis cover $/ 10.2307 / 3391946$ ?uid $=7751112$ \&uid $=3737664 \&$ uid $=212$ 9\&uid $=5909624 \&$ uid $=2$ \&uid $=70 \&$ uid $=3 \&$ uid $=67 \&$ uid $=62 \&$ uid $=47157 \&$ sid $=21101968559601>$. Acesso em: 5 nov 2011. doi: $10.2307 / 3391946$

SODERSTROM, T.R. et al. Distribution patterns of neotropical bamboos. In: VANZOLINI, P.E.; HEYER, W.R. (Eds.). Proceedings of a workshop on neotropical distribution patterns. Rio de Janeiro: Academia Brasileira de Ciências, 1998. p.121-157.

WANG, W. et al. Seed germination and seedling growth in the arrow bamboo Fargesia qinlingensis. Ecological Society of Japan, Japan, v.22, n.3, p.467-474, 2007.

WATSON, L.; DALLWITZ, M.J. The grass genera of the world: descriptions, illustrations, identification. Disponível em: $<$ http:// delta-intkey.com>. Acesso em: out. 2011.

WIDMER, Y. Flowering phenology of Chusquea bamboo with special to Chusquea talamancensis in Costa Rica. Journal of the American Bamboo Society, Lousiania, v.1, n.2, p.1-20, 1998. 